(Aus der Königl. Universitäts-Frauenklinik Erlangen. Früherer Director: Prof. Dr. Jung.)

\title{
Experimenteller Beitrag zur Frage der ascendirenden weiblichen Genitaltuberculose.
}

Von

\author{
Dr. med. Ernst Engelhorn.
}

(Mit 1 Textfigur.)

In der früher lebhaft umstrittenen Frage über die Entstehung und Verbreitung der Genitaltuberculose hat sich die Mehrzahl der Autoren dahin entschieden, dass die descendirende (secundäre, hämatogene) Form weitaus häufiger vorkommt, während die ascendirende (primäre) Genitaltuberculose theoretisch wohl möglich, aber überaus selten sei und deshalb praktisch wohl kaum in Betracht kommt.

So kam $\mathrm{Blau}^{1}$ ), auf dessen ausführliche Zusammenstellung über die bisher erschienenen Arbeiten ich hier verweise, auf Grund klinischer Beobachtung und eigener an Meerschweinchen angestellten Versuche zu folgendem Schluss: ein Ascendiren der Tuberculose in dem Sinne, dass Tuberkelbacillen sich dem Secretstrom entgegen nach aufwärts bewegten und dadurch auch in höher gelegenen Genitaltracten tuberculöse Processe erzeugten, liess sich nicht nur in keinem unserer klinischen Fälle, sondern auch in keinem unserer experimentellen Fälle beweisen.

Bei Fällen scheinbaren Ascendirens war es nicht auszuschliessen, dass es sich um gleichzeitige Infection höher gelegener Schleimhautparthien handelte oder um ein Wiedereinbrechen des Processes von aussen her in die Schleimhaut. Blau's Resultate stehen in diametralem Gegensatz zu denen von Jung und Bennecke, die auf Grund ihrer am Kaninchen vorgenommenen Versuche, in denen

1) Ueber die Entstehung und Verbreitung der Tuberculose im weiblichen Genitaltracte. Berlin 1909. 
776 Engelhorn, Zur Frage der ascendirenden weiblichen Genitaltuberculose.

sie eine aufsteigende Tuberculose erzeugen konnten, auch beim Menschen ein häufiges Vorkommen ascendirender Genitaltuberculose annehmen. Diese Resultate versuchten v. Baumgarten und seine Schüler durch eine Störung des normal nach abwärts gerichteten Secretstromes zu erklären. Weitere Versuche Jung's. die die Annahme einer ascendirenden Genitaltuberculose beweisen dürften, werden in nächster Zeit veröffentlicht werden. Bei dem wissenschaftlichen Interesse, das diese Frage auch heute noch beanspruchen darf, lag es nahe die Frage zu stellen, ob bei weiblichen Thieren (Kaninchen) experimentell der Nachweis erbracht werden kann, dass kleine in die Scheide gebrachte Elemente ohne Eigenbewegung and ohne infectiöse Eigenschaftern spontan in höher gelegene Abschnitte hinauf wandern. Diese Frage hat Kolde in seiner aus der Erlanger Fravenklinik erschienenen Inaugural-Dissertation an der Hand einiger am Kaninchen angestellter Experimente bejaht. Er verwandte zu seinen Versuchen feinst pulverisirtes Carmin, das er den Thieren in die Scheide brachte; er konnte später Carminkörnchen im Mesometrium nachweișen und schliesst daraus, dass die Carminkörnchen in der Scheide resorbirt worden sind und gemäss dem Verlauf der Lymphbahnen in die höher gelegenen Abschnitte des Geschlechtsapparates verschleppt wurden. Er konnte bei seinen Versuchen die von Baumgarten angenommene Secretstörung mit Sicherheit ausschliessen. Die Versuche von Kolde gaben mir zu weiteren Veranlassung.

Meine Versuchsauordnung war unter Benutzung der von $\mathrm{K}_{0} \mathrm{lde}$ gemachten Erfahrungen folgende: Einem weiblichen Kaninchen wurde feinst pulverisirtes Carmin, das in eine kleine Cacaobutterkugel verarbeitet war, auf einer langen Sonde in die Scheide vor die Portiones vaginales hinein gebracht. Um sicher in die höher gelegenen Parthien der Scheide und nicht durch die in die Scheide einmündende Urethra in die Harnblase zu gelangen, ist eine Controle der Sonde von der Bauchhöhle aus dringend nöthig. Der Versuch gestaltet sich kurz folgendermaassen: In Aethernarkose Laparotomie, Hervorziehen des Uterus. Unter Controle mit dem Finger wird die mit der Carmincacaobutterkugel armirte Sonde boch in die Scheide hinaufgeführt, hierauf wird der Uterus reponirt, die Bauchdecken werden durch Naht geschlossen. Inzwischen hat sich der kleine Globulus mit dem Carmin in der Scheide aufgelöst, man zieht hierauf die Sonde zurück. Diese Art des Verfahrens hat folgende Vorzüge: Mit Sicherheit lässt sich vermeiden, 
Engelhorn, Zur Frage der ascendirenden weiblichen Genitaltuberculose. 777 dass Carminpartikelchen schon beim Versuch höher hinauf als in die Scheide kommen (wie z. B. dann, wenn mit der Spritze eine Carminlösung injicirt wird); ferner wird vermieden, dass am Genitale eine Wunde gesetzt wird, durch die Carmintheilchen auf dem Lymphwege innerhalb des Organgewebes hinaufwandern können. Die Thiere wurden nach dem Versuch selbstverständlich vom Bock getrennt um eine etwaige Ascendirung mit dem Sperma mit Sicherheit ausschliessen zu können.

Nach 1-15 Tagen wurden die Thiere mit Chloroform getödtet und der ganze Genitalapparat in toto in aufstejgendem Alkohol gehärtet. Nach genügender Härtung wurden mehrfache Querschnitte in folgender Anordnung angelegt.

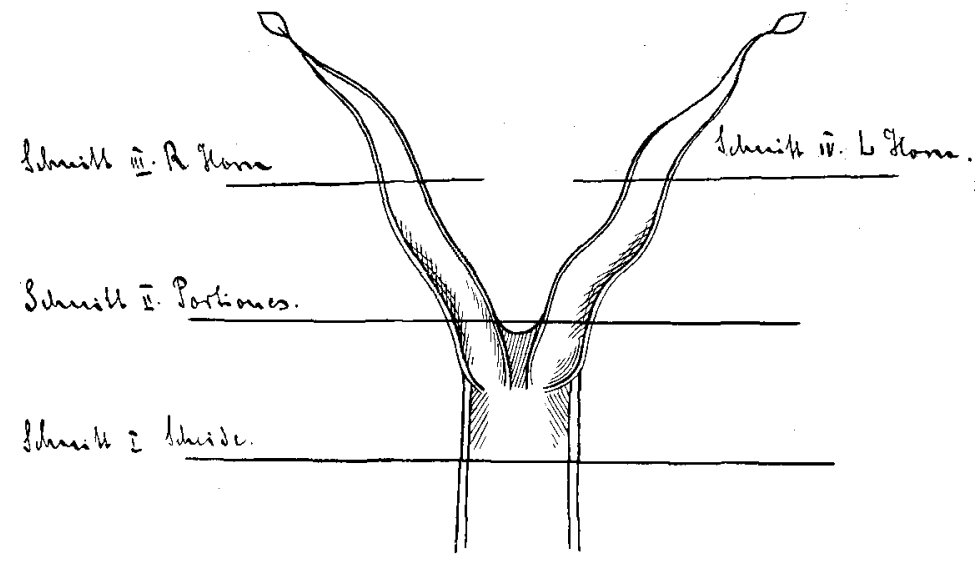

Die ausgeschnittenen Schnitte wurden in Xylolparalfin wciter behandelt, die 5-10 $\mu$ dicken Schnitte werden direct auf den Objectträger gebracht und nach Entfernung des Paraffins in Canadabalsam eingebeitet. Das Carmin ist nun deutlich in den ungefärbten Schnitten als feinste rothe Körnchen zu erkennen, hauptsächlich bei leicht ahgeblendetem Gesichtsfeld. Verwechselungen mit zum Präparat nicht gehörenden Fremdkörpern (Staub u.s.w.) sind ausgeschlossen, wie zum Beispiel bei der Anwendung von Tusche. Die folgende 'Tabelle giebt eine Uebersicht über meine Befunde.

Aus dieser Zusammenstellung geht hervor, dass in 11 von 17 Versuchsthieren das in der Scheide deponirte Carmin spontan nach oben gewandert ist und zwar auf dem Schleimhautwege. Bei den am 1., 2. und 3. Tag getödteten Thieren (A, B, D) 
778 Engelhorn, Zur Frage der ascendirenden weiblichen Genitaltuberculose.

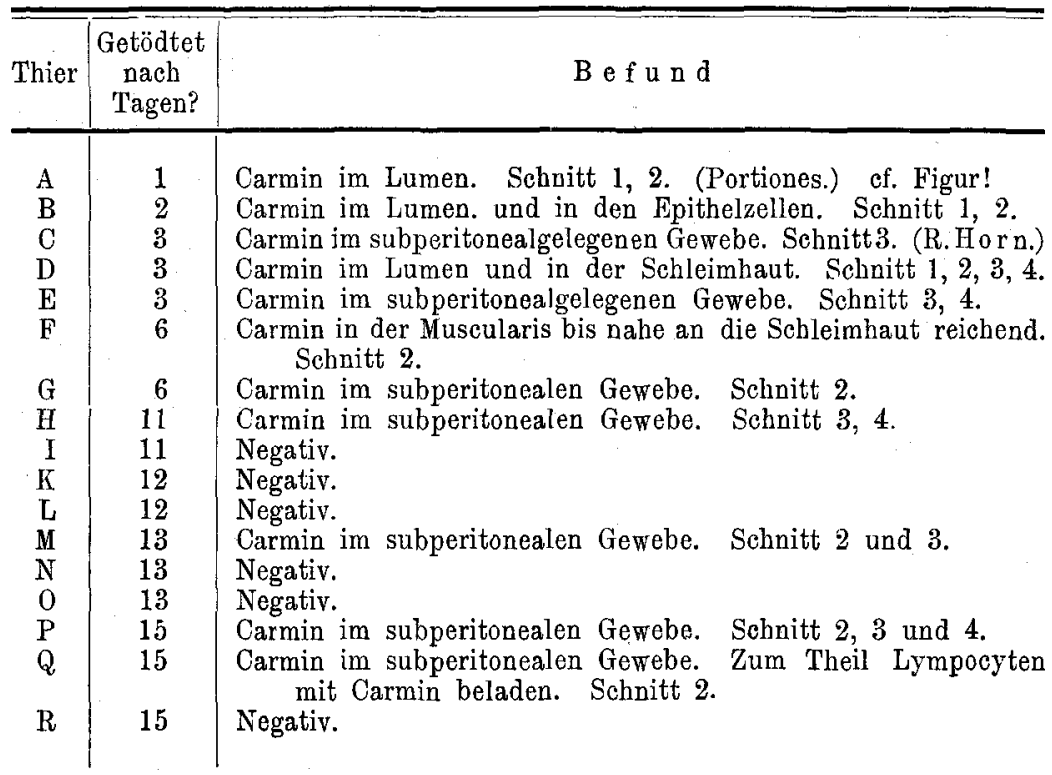

war das Carmin im Lumen und in dem Epithel der Schleimhaut mit Sicherheit nachzuweisen. Bei Thier D in allen Schnitten (1. 2. 3. 4.). Bei den später getödteten Thieren konnte im Lumen kein Carmin mehr nachgewiesen werden, sondern in der Muscularis und in den subperitoneal gelegenen Gewebsspalten. Bei Thier F reichten die Carminkörner noch ganz nahe an die Schleimhaut heran, in den anderen Präparaten waren sie dem Peritoneum mehr genähert.

Wir finden also in den ersten Tagen das Carmin im Lumen selbst; allmählich wird es durch die Schleimhaut nach der Muscularis und den subperitoneal gelegenen Lymphspalten weitergeschafft.

Von allen Schnitten wurden einige zur Controlle mit Hämatoxylin-Eosin gefärbt, um das genaue histologische Verhalten der Epithelzellen nachweisen zu, können. In keinem Präparat war eine Secretstauung oder eine Infiltration des Epithels nachzuweisen. Die Carminpartikel sind also entgegen dem normalen Secretstrom emporgewandert. Veranlasst wurde dieses Aufsteigen wohl durch antiperistaltische Bewegungen der Uterusmusculatur, die sowohl Kurdinowsky ${ }^{1}$ ) bei seinen Versuchen nachgewiesen hat, als auch Kolde und ich sie nach Eröffnung des Abdomens beinahe regelmässig am Kaninchenuterus bemerken konnten.

1) Dieses Archiv. Bd. 76 . 
Engelh orn, Zur Frage der ascendirenden weiblichen Genitaltuberculose. 779

Ebenso gut wie Körper ohne Eigenbewegung und ohne infectiöse Eigenschaften spontan von der Scheide nach höher gelegenen Genitalabschnitten hinaufwandern können, ebenso können wir uns ein Ascendiren des in die Kaninchenscheide gebrachten tuberculösen Materials denken.

Durch meine Versuche finden die oben erwähnten von Jung und Bennecke und hauptsächlich die demnächst zur Veröffentlichung gelangenden von Jung eine Stütze. Wie wir gesehen haben, sind nur in den allerersten Tagen Carminkörner im Lumen selbst nachzuweisen, vom 6. Tage ab finden sie sich im Myometrium und bleiben dort verhältnissmässig lange. Wir können es uns also denken, dass der Tuberkelbacillus von der Scheide durch das Lumen hinauf wandert und durch die Schleimhaut in das Myometrium eindringt. Es kann also der Tuberkelbacillus beim Hinaufwandern zuerst in der Schleimhaut des Uterus selbst tuberculöse Veränderungen hervorrufen, oder es ist denkbar, dass er die Schleimhaut durchdringt, ohne in ihr Veränderungen hervorgerufen zu haben, und der tuberculöse Process sich im Myometrium entwickelt und von dort aus nach der Schleimhaut zurückwächst.

Inwieweit diese Verhältnisse auf den Menschen übertragen werden dürfen, darüber können einstweilen nur vorsichtige Schlüsse gezogen werden (Jung).

Von manchen Autoren wird dem Scheidensecret eine baktericide Kraft dem Tuberkelbacillus gegenüber angenommen (Menge und Krönig, Will). Ich habe diese Frage durch folgende Versuche nachgeprüft. Es wurde mehreren Schwangeren Scheidensecret entnommen und diese Scheidensecrete zu einem Gemische vermengt. In dieses Gemisch, dessen Reaction jedesmal geprüft wurde und das ich stets sauer gefunden habe, wurden nun Tuberkelbacillen eingesät und von dieser Mischung (Tuberkelbacillen und Secret) sofort nach der Einsaat und dann nach 1, 3, 6, 12-24 Stunden je ein Cubikcentimeter einem Meerschweinchen intraperitoneal eingespritat. Die Einsaat des Tuberkelbacillus wurde so gewählt, dass auf einen Cubikcentimeter Mischung je eine $1 / 2$ Normal-Oese unserer Tuberculosecultur (Typus humanus) kam, die an und für sich eben hinreichte, um ein Thier nach 4-6 Wochen zum Exitus zu bringen.

Sämmtliche von mir geimpften Thiere (14) erlagen ihrer Tuberculose, sodass wir eine baktericide Kraft des Scheidensecrets auf die Tuberkelbacillen ausschliessen zu dürfen glauben. 
780 Engelhorn, Zur Frage der ascendirenden weiblichen Genitaltuberculose.

Wenn wir hiernach dem Scheidensecret eine baktericide Kraft auf den Tuberkelbacillus absprechen dürfen und wenn Tuberkelbacillen, wenn auch selten, bei ganz gesunden Frauen in der Scheide gefunden werden [Schöttke $\left.\left.\mathrm{e}^{1}\right)\right]$, können wir uns auch beim menschlichen Weibe ein Ascendiren der Keime auf dem Schleimhautwege vorstellen und sind berechtigt an der Lehre von der ascendirenden Genitaltuberculose festzuhalten.

1) Inang.-Diss. Greifswald 1907. 